\title{
Macrophage PD-L1 strikes back: PD-1/PD-L1 interaction drives macrophages toward regulatory subsets
}

\author{
Yun-Jung Lee, Young-Hye Moon, Kyeong Eun Hyung, Jong-Sun Yoo, Mi Ji Lee, Ik Hee Lee, \\ Byung Sung Go, Kwang Woo Hwang*
}

Laboratory of Host Defense Modulation, College of Pharmacy, Chung-Ang University, Seoul, Korea

Email: ${ }^{*}$ khwang@cau.ac.kr

Received 21 May 2013; revised 25 June 2013; accepted 19 July 2013

Copyright (C) 2013 Yun-Jung Lee et al. This is an open access article distributed under the Creative Commons Attribution License, which permits unrestricted use, distribution, and reproduction in any medium, provided the original work is properly cited.

\section{ABSTRACT}

Activated macrophages have been simply defined as cells that secrete inflammatory mediators and kill intracellular pathogens until few years ago. Recent studies have proposed a new classification system to separate activated macrophages based on their functional phenotypes: host defense, wound healing, and immune regulation. Regulatory macrophages can arise following innate or adaptive immune responses and hinder macrophage-mediated host defense and inflammatory functions by inhibiting the production of pro-inflammatory mediators. In this study, we investigated whether PD-1 and PD-L1 interaction between macrophages and $T$ cells alters macrophage activities. Our data provide evidence for PD-1/PD-L1 engagement inducing a regulatory profile in macrophages. Regulatory macrophages derived from PDL1 signaling lost their host defense activity, which consists of the production of pro-inflammatory cytokine IL-6 and the exhibition of increased IL-10, SPHK1 and LIGHT gene levels in early phases of LPS stimulation. This differentiation seems to occur through excessive activation of TLR4 downstream MAPK signaling pathways. Regulatory macrophages induced from PD-1/PD-L1 interaction decrease inflammatory mediators and produce anti-inflammatory cytokines, so this macrophage subset has been under considerable attention as a possible immune regulation mechanism. Understanding and modulating regulatory macrophages may lead to new approches to treat or prevent auto-immune diseases such as type I diabetes, rheumatic syndrome and hypersensitivity-related diseases, which are concerned with the overproduction of inflammatory cytokines in macroages.

"Corresponding author.
Keywords: Regulatory Macrophage; PD-1; PD-L1

\section{INTRODUCTION}

Macrophages are innate immune cells, and are the first line of defense against invading pathogens [1], and they are also antigen presenting cells (APCs), participating in adaptive immunity. Macrophages have versatile abilities, including phagocytosis, antigen presentation, antimicrobial toxicity, and tissue remodeling as well as the secretion of a wide range of growth factors, cytokines, complement components, prostaglandins, and enzymes [2]. Monocytes developed from hematopoietic cells start to circulate blood and enter tissues [3] and depend on the tissue environment they are capable of realizing different physiological functions [4]. The signature role of macrophages in host defense is the production of inflammatory cytokines IL- $1 \beta$, IL- 6 , TNF- $\alpha$ and nitric oxide (NO). In addition to this, macrophages can be activated alternatively and exhibit wound repair and immune regulation functions [5].

A recent system of classification was proposed by Mosser et al. in 2008 to separate activated macrophages based on their functional phenotypes: host defense, wound healing, and immune regulation [6]. In a similar review, they also emphasize the importance of understanding that macrophages exhibit not only the three currently recognized states of activation, but a broad spectrum of activation states [7]. Since macrophages have high plasticity [8], they are not restricted to a single activation state; there may be distinct macrophage states which exhibit characteristics of two or more activation states, termed "hybrid" macrophages [7].

Activation of antigen-specific $\mathrm{T}$ cells is dependent on $\mathrm{T}$ cell receptor (TCR) and major-histocompatibility-complex (MHC) interactions, while crucial additional costimulatory signals are also necessary. In the absence of 
costimulatory signals, lymphocytes fail to respond effectively and are rendered anergy [9]. These signals are delivered to $T$ cells by costimulatory cell surface molecules expressed on APCs. The best characterized positive costimulatory receptor on $\mathrm{T}$ cells is $\mathrm{CD} 28$, which binds B7-1/B7-2 and is mostly expressed on APCs and activated $\mathrm{T}$ cells. This concurrent engagement during TCR ligation is necessary for $\mathrm{T}$ cell activation and $\mathrm{B} 7-1 / \mathrm{B} 7-2$ and cytotoxic $\mathrm{T}$ lymphocyte attenuator-4 (CTLA-4) interaction is needed for tolerance. Alongside this, Programmed death 1 (PD-1) receptor on $\mathrm{T}$ cells and its ligands PD-L1 and PD-L2 on APCs interactions also play an important role in T cell activity regulation [10].

Programmed death 1 (PD-1) is an immunoreceptor of the CD28/CTLA-4 family whose expression is induced in activated $\mathrm{T}$ and $\mathrm{B}$ cells and in macrophages [11]. PD-1 is a 50 - $55 \mathrm{kDa}$ type I transmembrane glycoprotein composed of an IgV-type extracellular domain sharing $21 \%$ $33 \%$ sequence identity with CTLA-4, CD28 and ICOS $[12,13]$. The broader expression of PD-1 contrasts with restricted expression of other CD28 family members to $\mathrm{T}$ cells, suggesting that PD-1 regulates a wider spectrum of immune response compared with other $\mathrm{CD} 28$ family members. PD-L1/PD-L2 are ligands known as bind to PD-1 [14], and share 20\% amino acid identities with B7-1/B7-2 that are ligands for CD28 and CTLA-4 [1517]. PD-L1 is constitutively expressed on T cells, B cells, macrophages and dendritic cells but PD-L2 expression is regulated more tightly; and it is observed on activated macrophages and DCs [18].

There are several genetically modified mouse models that alter PD-1 expression. PD-1 deficient (PD-1-/-) mice were generated by deletion of the transmembrane and cytoplasmic domain exons, and some studies show the inhibitory role of PD-1 in vivo using this mouse model [19]. PD-1-/- mice exhibit an increase in double positive $\mathrm{T}$ cells, but a reduced number of single-positive CD8 T cells, suggesting a role for this pathway in setting thresholds for thymocyte differentations [20]. PD-1-/- mice develop various autoimmune diseases depending on their genetic background. Using NOD-PD-1-/- mice as an efficient animal model of type 1 diabetes, diabetes-susceptible loci were screened by genetic linkage analysis. On the other hand, PD-1 transgenic mice, which were engineered in our laboratory, constitutively express $\mathrm{T}$ cell-restricted PD-1 under the control of the Lck proximal promoter and CD2 locus control. PD-1 Tg mice did not develop gross abnormalities of thymic development and displayed a normal number of thymocyte subsets and peripheral T cells. In vitro, PD-1 Tg T cells had reduced function upon TCR stimulation and cross-linking of PD-1 resulted in diminished phosphorylation of protein kinase C- $\theta$ and Akt, as well as increased activation of the phosphate and tensin homology [20].
It has been established that costimulatory molecules such as B7-1/B7-2 on APCs interacting with CD28/ CTLA-4 on T cells regulate the T cell activation of tolerance through a unidirectional signal pathway, APCs to $\mathrm{T}$ cells [21-23]. However, recent studies have shown that these signals are not limited to $\mathrm{T}$ cell regulation, but also modulate APCs through costimulatory ligands B7-1/B7-2. Bidirectional signaling along the B7-CTLA-4 coreceptor pathway enables reciprocal conditioning of $\mathrm{T}$ cells and dendritic cells. T cells can instruct dendritic cells to manifest tolerogenic properties after CTLA-4 engagement to B7. In this manner, IDO expression is up-regulated after engagement by CTLA-4 [8]. A separate study reported that B7-CD28 engagement induces IL-6, IFN- $\gamma$ production through the p38 MAPK signal pathway in DCs [24].

Preceding research performed using PD-1 Tg mice showed that inhibitory effects of PD-1 prevent the induction of type 1 diabetes and these results were driven by changes in $\mathrm{T}$ cell function. Contrary to expectations, while the number of $\mathrm{T}$ cells remained unchanged, the number of macrophages sharply decreased [25]. This altered macrophage population is induced by decreased IFN- $\gamma$ secretion from PD-1 Tg T cell or, surprisingly, it may be due to changes in the macrophage itself; thus the possibility of overexpressed PD-1 on T cells acting on macrophages came into the spotlight. To investigate whether the interaction between PD-1 and its ligands alters macrophage activities, macrophages were stimulated with PD-1 human Ig or anti PD-L1 antibody. We found these engagements increase SPHK1, LIGHT, IL10 and decrease IL-6 in early phase stimulation. These results suggest that signal transduction through PD-L1 drives macrophages toward regulatory subsets.

\section{MATERIALS AND METHODS}

\subsection{Cell Culture}

RAW 264.7 macrophage cell line was obtained from the American Type Culture Collection (ATCC) and maintained in DMEM medium (Cellgro) supplemented with $10 \%$ heat-inactivated FBS (Fetal bovine serum, Cellgro), $2 \mathrm{mM}$ L-glutamine, $100 \mathrm{U} / \mathrm{ml}$ penicillin and streptomycin (Cellgro) at $37^{\circ} \mathrm{C}$ in a $5 \% \mathrm{CO}_{2}$ humidified incubator.

\subsection{Mice}

Wild type male C57BL/6 mice, aged 6 to 12 weeks, were purchased from Orient Bio as controls, and PD-1 transgenic mice, which overexpress PD-1, particularly on $\mathrm{T}$ cells using the lck promoter, were generated by professor Kwang Woo Hwang.

Animals were maintained under the controlled conditions of temperature $\left(21^{\circ} \mathrm{C} \pm 3^{\circ} \mathrm{C}\right)$, relative humidity 
$(50 \% \pm 10 \%)$, and light (10 and 14 hours of light and dark, respectively) in a specific pathogen-free environment.

\subsection{Reagents}

Lipopolysaccharide (LPS, Sigma) was used to activate RAW 264.7 macrophage cell lines at $1 \mu \mathrm{g} / \mathrm{ml}$ of working concentration. Human Ig and PD-1 human Ig fusion proteins were constructed in our laboratory using antibody purification from $\mathrm{pFUSE}$ human $\operatorname{IgG}_{1} \mathrm{Fc}$ empty vector or PD-1 extracellular domain inserted pFUSE human $\operatorname{IgG}_{1}$ Fc vector transfected $\mathrm{CHO}$ cell. Purified rat $\mathrm{IgG}_{2}$ and anti PD-L1 antibody (clone: MIH5) was purchased from ebioscience.

\subsection{Beads Conjugation}

$25 \mu \mathrm{l}\left(1 \times 10^{7}\right)$ of M-450 Tosyl activated Dynabeads (Invitrogen) were conjugated with $5 \mu \mathrm{g}$ of human Ig, PD-1 human Ig, rat IgG2a and anti PD-L1 antibody at $37^{\circ} \mathrm{C}$ for 16 hours with gentle rolling. After ligation step, the antibody was incubated with blocking buffer $(0.2 \mathrm{M}$ Tris w/ $0.1 \% \mathrm{BSA}, \mathrm{pH} 8.5$ ) at $37^{\circ} \mathrm{C}$ for 4 hours to deactivate the remaining tosyl group and resuspended in culture media at working concentration.

\subsection{Real-Time PCR}

$1.2 \times 10^{6}$ RAW 264.7 cells were plated in a 24-well multi plate and, after 6 hours, $10 \mu \mathrm{g} / \mathrm{ml}$ of beads conjugated with human Ig and/or PD-1 human Ig or $10 \mu \mathrm{g} / \mathrm{ml}$ and 20 $\mu \mathrm{g} / \mathrm{ml}$ of beads conjugated with rat IgG and anti PD-L1 antibody were treated to each groups for 4 hours followed by $1 \mu \mathrm{g} / \mathrm{ml}$ of LPS treatment. After 1 hour and 30 min, total RNA was isolated from each sample using Trizol reagent (Invitrogen). RNA was transcribed to cDNA at $42^{\circ} \mathrm{C}$ for 1 hour in a volume of $25 \mu$ containing $5 \times$ RT buffer, $10 \mathrm{mM}$ dNTPs 200 units Maloney murine leukemia virus reverse transcriptase and 100 pmole of oligo-dT primer.

The cDNA was then used for PCR with $2 \times$ iQTM SYBR Green Supermix (Bio-Rad) to determine the mRNA level of SPHK1, LIGHT, IL-6, IL-10 and GAPDH. Amplification was performed under the following conditions: $95^{\circ} \mathrm{C}$ for $5 \mathrm{~min}, 45$ cycles of $95^{\circ} \mathrm{C}$ for $20 \mathrm{sec}$ and $60^{\circ} \mathrm{C}$ for $30 \mathrm{sec}$, followed by a final extension of $55^{\circ} \mathrm{C}$ for 1 min using an iQ5TM Thermal Cycler (Bio$\mathrm{Rad})$. Gene expression was normalized to the expression of GAPDH.

\subsection{Enzyme-Linked Immunosorbent Assay (ELISA)}

$2 \times 10^{5}$ RAW 264.7 cells were plated in a 96-well multi plate and, after 6 hours, $10 \mu \mathrm{g} / \mathrm{ml}$ of beads conjugated with human Ig and/or PD-1 human Ig or $10 \mu \mathrm{g} / \mathrm{ml}$ and 20 $\mu \mathrm{g} / \mathrm{ml}$ of beads conjugated with rat IgG and anti PD-L1 antibody were treated for 4 hours followed by $1 \mu \mathrm{g} / \mathrm{ml}$ of LPS treatment. Cell free supernatants were harvested after 16 hours, and the concentration of IL-10, IL- 6 and TNF in each sample was detected by ELISA.

\subsection{NO Assay}

$2 \times 10^{5}$ RAW 264.7 cells were plated in a 96-well multi plate and after 6 hours, $10 \mu \mathrm{g} / \mathrm{ml}$ of beads conjugated with human Ig and/or PD-1 human Ig or $10 \mu \mathrm{g} / \mathrm{ml}$ and 20 $\mu \mathrm{g} / \mathrm{ml}$ of beads conjugated with rat IgG and anti PD-L1 antibody were treated for 4 hours followed by $1 \mu \mathrm{g} / \mathrm{ml}$ of LPS treatment. After 16 hours, cell free supernatants were assessed for nitric oxide production. Each supernatant was mixed with an equal volume of Griess reagent (Sigma). The absorbance of the mixture was determined at $540 \mathrm{~nm}$ using a microplate reader, and nitrite concentration was determined using a dilution of standard nitrite.

\subsection{Western Blot Analysis}

$1.2 \times 10^{6}$ RAW 264.7 cells were plated in a 6-well multi plate and after 6 hours, $10 \mu \mathrm{g} / \mathrm{ml}$ of beads conjugated with human Ig and/or PD-1 human Ig or $10 \mu \mathrm{g} / \mathrm{ml}$ and 20 $\mu \mathrm{g} / \mathrm{ml}$ of beads conjugated with rat IgG and anti PD-L1 antibody were treated for 4 hours followed by $1 \mu \mathrm{g} / \mathrm{ml}$ of LPS treatment.

After $30 \mathrm{~min}$, whole cell proteins were extracted in RIPA buffer (Elpis Biotech) and equivalent amounts of proteins were separated by SDS-PAGE, transferred to polyvinylidene fluoride (PVDF) membranes and probed with antibodies against $\mathrm{p}-\mathrm{I} \kappa \mathrm{B} \alpha, \mathrm{I} \kappa \mathrm{B} \alpha, p$-ERK, ERK, $\mathrm{p}-\mathrm{p} 38, \mathrm{p} 38, \mathrm{p}-J \mathrm{NK}, J N K$ and $\beta$-actin (Cell Signaling). The membranes were then incubated with anti-mouse IgG or anti-rabbit IgG HRP-linked antibodies (Cell Signaling), and developed using the BioFX (SurModics) by chemiluminescence (Bio-Rad Chemidoc system) and analyzed by Quantity One (Bio-Rad).

\subsection{Mitomycin C Treatment}

PD-1 transgenic mice and wild type mice were sacrificed and splenocytes were isolated. To purify $\mathrm{T}$ cells, splenocytes were incubated with beads-conjugated anti Thy 1.2 antibody for $15 \mathrm{~min}$ on ice and then T cells were purified using MACS cell separation (Miltenyi Biotech). Splenocytes or T cells were incubated with $75 \mu \mathrm{g} / \mathrm{ml}$ of mitomycin $\mathrm{C}$ for $30 \mathrm{~min}$ at $37^{\circ} \mathrm{C}$ and washed with media several times.

\subsection{Flow Cytometric Analysis}

For extracellular staining of surface molecules on macrophages, RAW cells were cocultured with mitomycin 
C-treated WT or PD-1 Tg splenocytes and were harvested and stained with FITC-conjugated anti CD11b antibody and PE-conjugated anti B7-1, B7-2, PD-L1 antibody (ebioscience). Stained cells were washed with PBS and analyzed by using FACSCalibur and CellQuest analysis software (BD Bioscience).

\subsection{Proliferation Assay (MTT Assay)}

RAW cells were cocultured with mitomycin C-treated WT or PD-1 Tg T cells for 24 hours. After culture, MTT [3-(4,5-dimethylthiazolyl-2)-2, 5-diphenyltetrazolium bromide] solution (Sigma) was added to each well and incubated for $2 \mathrm{~h}$. An equal volume of solubilization solution $(0.04 \mathrm{~N} \mathrm{HCl}$ in isopropanol) was added to each well and evaluated at $570 \mathrm{~nm}$ wavelength.

\subsection{Statistical Analysis}

Data are expressed as mean $\pm \mathrm{SD}$, and statistical analyses were performed by using a Student's $t$ test. Values were considered statistically significant with ${ }^{*}, p<0.05 ;{ }^{* *}, p<$ 0.01 ; and ${ }^{* * *}, p<0.001$.

\section{RESULTS}

\subsection{Possible Role of PD-1/PD-L1 Engagement in Macrophage Activity Modulation}

The interaction between PD-1 expressed on activated T cells and PD-L1 on APCs delivers tolerogenic signals to $\mathrm{T}$ cells, resulting in the decrease of IL- 2 , IFN- $\gamma$ production, cell viability and induce $\mathrm{T}$ cell tolerance in general. On the contrary to this standard signaling, we focused on the possible two-way signal transduction in PD-1/PD-L1 engagement. B7-1/B7-2 ligands are commonly known as molecules which deliver costimulatory signals to receptors on other cells, especially $\mathrm{T}$ cells. However, recent studies have reported that $\mathrm{B} 7-1 / \mathrm{B} 7-2$ can receive reciprocal signals by the engagement of these ligands to CTLA-4 and CD28. For instance, CTLA-4 Ig was found to regulate dendritic cells using IDO expression and CD28 Ig was shown to enhance IL-6, IFN- $\gamma$ production in dendritic cells $[19,26]$.

Similar to how CTLA-4/CD28 and B7 regulates dendritic cells through B7-1/B7-2 signal transduction, PD-1/ PD-L1 interaction may be the key factor in controlling macrophage activity. In the in vitro portion of this study, the mouse macrophage cell line RAW264.7 was used. There are two ligands for PD-1, PD-L1 and PD-L2 on macrophages. To elucidate which ligand is more appropriate for this study, RAW 264.7 cells were incubated in the presence of LPS for stimulation and, after 24 hours, FACS analysis was performed (data not shown). As a result, PD-L1 expression was significantly higher in LPS-treated RAW 264.7 cells but PD-L2 was not altered and was barely expressed. In order to stimulate PD-L1 on macrophages, both anti PD-L1 antibody and PD-1 human Ig, constructed in our laboratory, were used.

\subsection{PD-1/PD-L1 Interaction Is Shown to Differentiate Macrophages toward Regulatory Subsets}

In contrast to classical activation in which macrophages are stimulated with TLR agonists such as LPS, differentiation into regulatory macrophages occurs when TLR stimulation is combined with several other stimuli [5]. These macrophages exhibit anti-inflammatory effects, with increased production of IL-10. Unlike host defense macrophages or wound healing macrophages, regulatory macrophages display up-regulation of SPHK1 and LIGHT.

We investigated in order to clarify which reaction is elicited in macrophages when given $\alpha$ PD-L1 antibody or PD-1 Ig with LPS stimulation. To visualize this phenomenon at the transcriptional level, RNA was isolated 1 hour $30 \mathrm{~min}$ after LPS treatment and relative gene expression was detected using real-time PCR (Figure 1).

In order to identify whether signals through PD-L1 change macrophage characteristics towards a regulatory subset, SPHK1 and LIGHT gene expression was evaluated. It has been reported that when IgG-immune complex binds with the Fcy receptor expressed on macrophages with TLR stimulation, these signals can induce macrophage differentiation toward regulatory subsets. As expected from preceding studies, isotype control rat IgG or human Ig also enhanced expression of these two genes. Nevertheless, analysis of these genes revealed a significant enhancement of LIGHT and SPHK1 by $\alpha$ PD-L1 antibody and PD-1 hIg compared to the isotype control. In Figure 1(b), while $10 \mu \mathrm{g} / \mathrm{ml}$ of $\alpha \mathrm{PD}-\mathrm{L} 1$ antibody treatment only increased SPHK1 and LIGHT expression similar to rat $\operatorname{IgG}, 20 \mu \mathrm{g} / \mathrm{ml}$ treatment up-regulated the expression of these genes compared to rat IgG. In addition, the IL-10 relative gene expression level was increased in LPS and PD-1 Ig groups rather than hIg groups and also increased in $20 \mu \mathrm{g} / \mathrm{ml}$ of $\alpha \mathrm{PD}-\mathrm{L} 1$ antibody group. IL-6 gene level was slightly reduced by $\alpha$ PD-L1 antibody, PD-1 Ig as compared to the isotype control and iNOS was detected at basal level in either group (data not shown).

Considering the differences in the expression of several genes in PD-L1 signaling, we investigated whether or not cytokine secretion is modulated when PD-L1 signaling is delivered into macrophages. RAW 264.7 cells were cultured with bead-conjugated hIg or PD-1 hIg (Figure 2(a)) and rat IgG or anti PD-L1 antibody (Figure 2(b)) along with LPS stimulation. ELISA analysis of culture supernatants, as well as gene expression in the same group, revealed a similar increasing or decreasing 

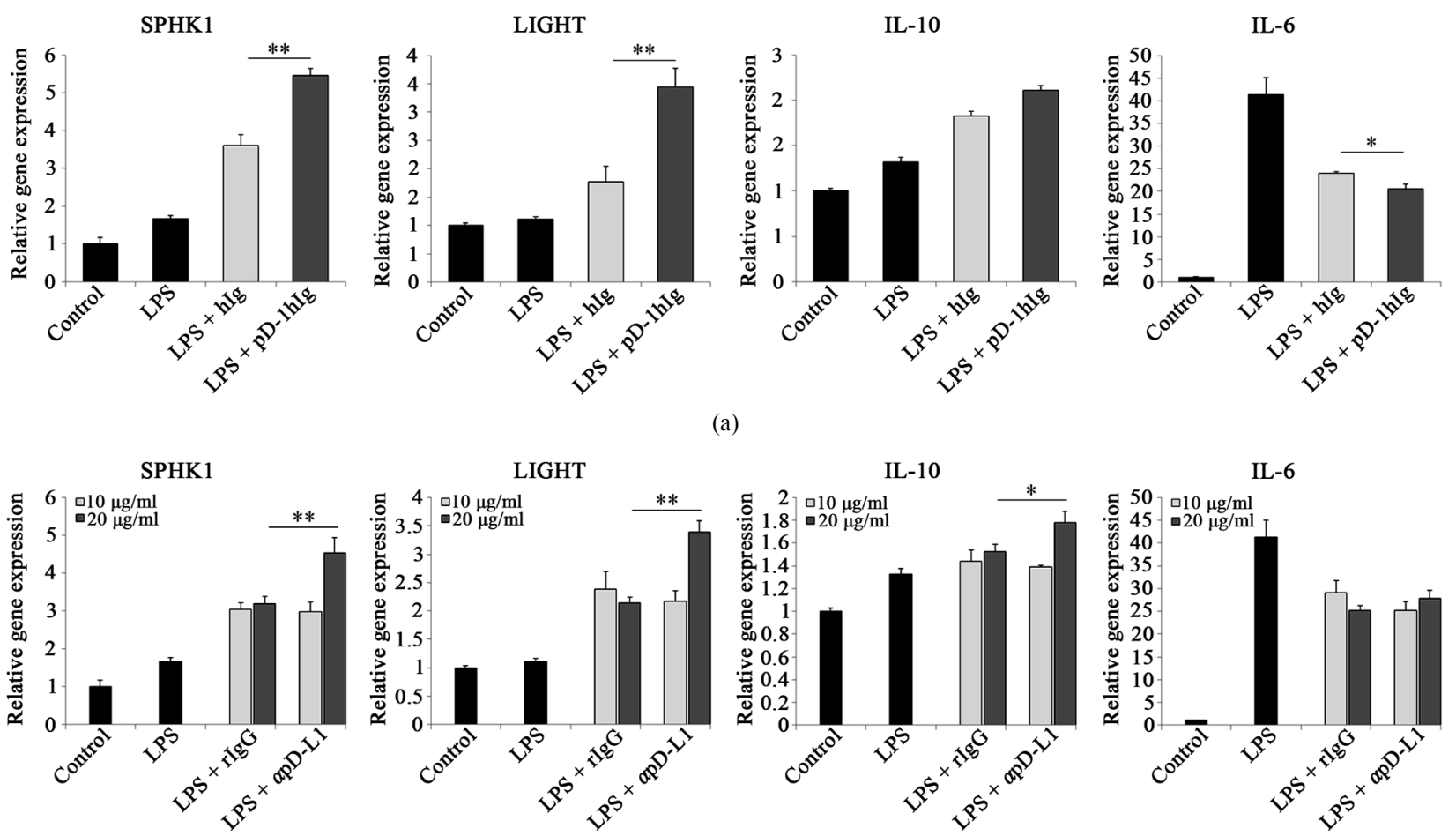

(b)

RAW 264.7 cells were treated with $10 \mu \mathrm{g} / \mathrm{ml}$ of beads conjugated with human Ig and $/$ or human $\mathrm{Ig}$ (a) or $10 \mu \mathrm{g} / \mathrm{ml} \mathrm{and} 20 \mu \mathrm{g} / \mathrm{ml}$ of beads conjugated with rat IgG and anti PD-L1 antibody (b) for 4 hours followed by $1 \mu \mathrm{g} / \mathrm{ml}$ of LPS treatment for 1 hour 30 min prior to mRNA extraction. SPHK1, LIGHT, IL-10 and IL-6 mRNA were verified through real-time PCR. Each gene level was relatively expressed vs. control cells and all gene quantities were normalized to the expression of GAPDH. Data are presented as mean \pm SD for triplicate determinations. Student's $t$ test; ${ }^{*} p<0.05 ;{ }^{* * *} p<0.01 ;$ and ${ }^{* * *} p<0.001$ vs. beads conjugated with human $\operatorname{Ig}(\mathrm{a})$ or rat $\operatorname{IgG}(\mathrm{b})$. All data are representative of at least three individual experiments.

Figure 1. Signal transductions through PD-L1 on macrophages modulate several cytokines and amplify LIGHT and SPHK1 at the gene level.

pattern. Anti-inflammatory cytokine IL-10 secretion was extremely more elevated by PD-1 hIg than by hIg, and also increased by treatment with $\alpha \mathrm{PD}-\mathrm{L} 1$ antibody in both $10 \mu \mathrm{g} / \mathrm{ml}$ and $20 \mu \mathrm{g} / \mathrm{ml}$ treatments. On the contrary, pro-inflammatory cytokine IL-6 secretion was decreased by PD-1 hIg and $\alpha$ PD-L1 antibody. However, TNF, NO and other pro-inflammatory mediators did not exhibit obvious differences by treatment with PD-1 hIg and $\alpha$ PD-L1 antibody in the presence of LPS stimulation compared to the isotype control or LPS stimulation alone.

These results suggest the possible role of PD-L1 signaling in the differentiation of macrophages into regulatory subsets.

\subsection{PD-1/PD-L1 Signal Pathway Associated with LPS-Induced MAPK Signaling Pathway}

Although little is known about the biomarkers of regulatory macrophages, a few have been suggested as markers, for instance IL-10, SPHK1 and LIGHT. However, the role of these factors and downstream signaling pathways remain poorly defined.

$\mathrm{PI} 3 \mathrm{~K}$ is an upstream molecule of NF- $\kappa \mathrm{B}$ signaling and
$\mathrm{I} \kappa \mathrm{B}$ is an $\mathrm{NF}-\kappa \mathrm{B}$ regulator that binds to NF- $\kappa \mathrm{B}$ and is phosphorylated and degraded when the NF- $\kappa \mathrm{B}$ signaling pathway is activated. Therefore, phosphorylation of $\mathrm{I} \kappa \mathrm{B}$ was evaluated to determine the activity of PI3K and NF- $\kappa$ B. Hence, the activity of four signaling molecules, $\mathrm{I} \kappa \mathrm{B}, \mathrm{ERK}, \mathrm{p} 38$ and JNK were visualized to clarify the complex signaling process involved in the differentiation of regulatory macrophages. To verify the involvement of known signal transduction pathways downstream of TLR4 and PD-L1 during differentiation, RAW 264.7 cells were treated with $1 \mu \mathrm{g} / \mathrm{ml}$ LPS in the presence of PD-1 hIg or $\alpha$ PD-L1 antibodies. After 30 min, LPS-induced MAPK signaling pathway molecules, NF- $\kappa \mathrm{B}$, ERK, p38 and JNK, were detected by western blotting (Figure 3). We assessed the activation of these signal molecules by detecting the phosphorylation of $\mathrm{I} \kappa \mathrm{B}$, a regulator of NF- $\kappa \mathrm{B}$ that binds to NF- $\kappa \mathrm{B}$ and is phosphorylated and degraded when the NF- $\kappa \mathrm{B}$ signaling pathway is activated, and the phosphorylation of ERK, p38 and JNK. As shown in Figures 3(a) and (b), PD-1 hIg and $\alpha \mathrm{PD}-\mathrm{L} 1$ antibody treatment enhanced the phosphorylation of $\mathrm{I} \kappa \mathrm{B}, \mathrm{ERK}, \mathrm{p} 38$ and JNK more than LPS stimulation alone. 
IL-10

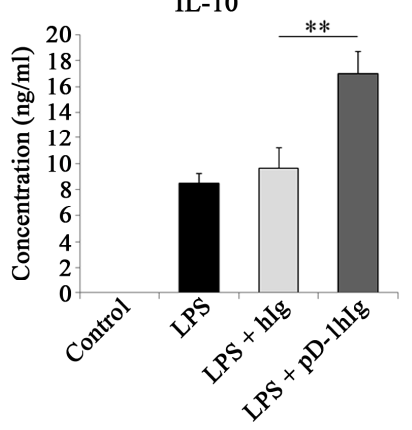

IL-10

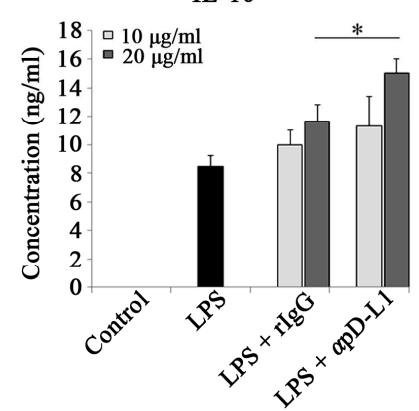

IL-6

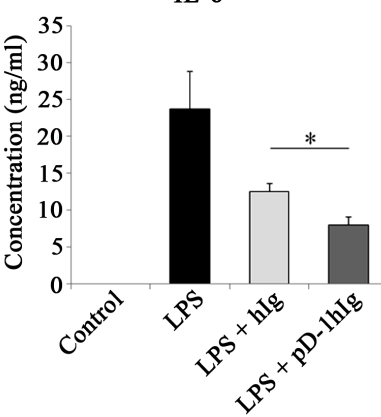

IL-6

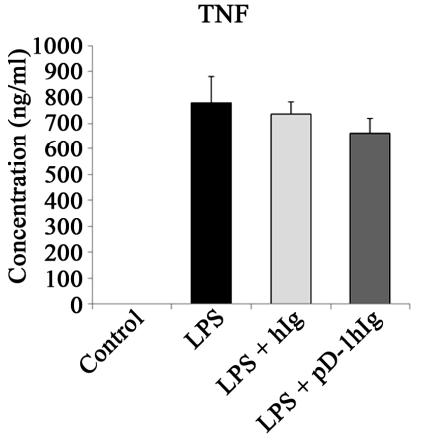

(a)

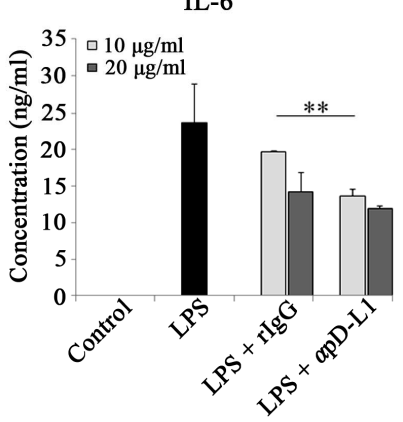

TNF

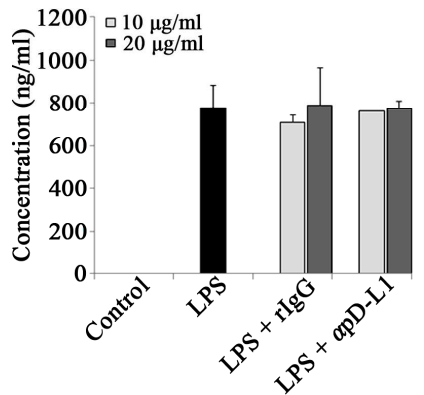

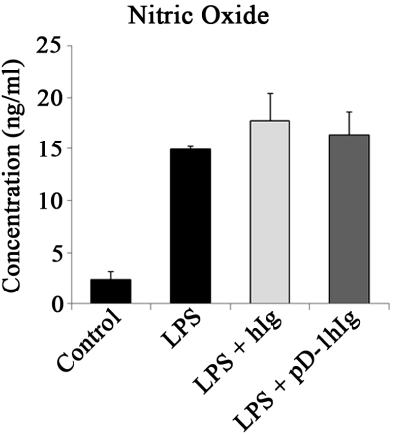

Nitric Oxide

(b)

RAW 264.7 cells were treated with $10 \mu \mathrm{g} / \mathrm{ml}$ of beads conjugated with human Ig and/or PD-1 human Ig (a) or $10 \mu \mathrm{g} / \mathrm{ml}$ and $20 \mu \mathrm{g} / \mathrm{ml}$ of beads conjugated with rat IgG and anti PD-L1 antibody (b) for 4 hours followed by $1 \mu \mathrm{g} / \mathrm{ml}$ of LPS treatment. After 16 hours, cell free supernatants were harvested and cytokine levels were detected by ELISA; nitric oxide concentration was detected using NO assay. Data are presented as mean \pm SD for triplicate determinations. Student's $t$ test; ${ }^{*} p<0.05 ;{ }^{* *} p<0.01$; and ${ }^{* * *} p<0.001$ vs. beads conjugated with human Ig (a) or rat IgG (b). All data are representative of at least three individual experiments.

Figure 2. PD-L1 signaling enhanced IL-10 production but decreased pro-inflammatory cytokine IL-6.

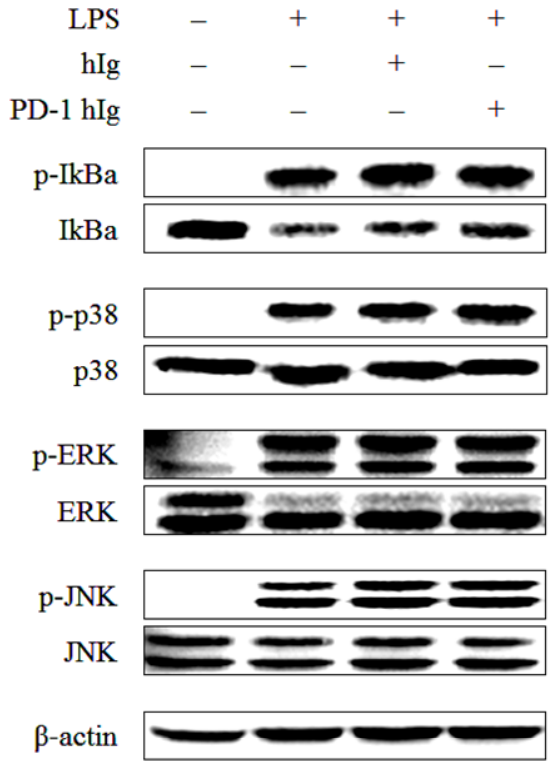

(a)

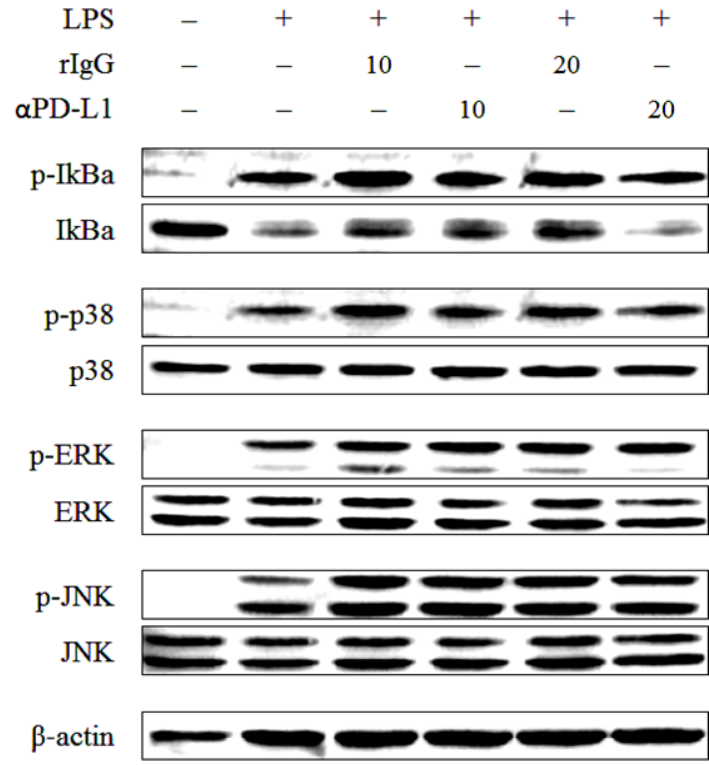

(b)

RAW 264.7 cells were treated with $10 \mu \mathrm{g} / \mathrm{ml}$ of beads conjugated with human Ig and/or PD-1 human Ig (a) or $10 \mu \mathrm{g} / \mathrm{ml}$ and $20 \mu \mathrm{g} / \mathrm{ml}$ of beads conjugated with rat IgG and anti PD-L1 antibody (b) for 4 hours followed by $1 \mu \mathrm{g} / \mathrm{ml}$ of LPS treatment for $30 \mathrm{~min}$. Then cell lysates were harvested, and western blot analysis was conducted using the antibodies for phospho-I $\kappa \mathrm{B}$, I $\kappa \mathrm{B}$, phospho-ERK, ERK, phospho-p38, p38, phospho-JNK and JNK. The detection of $\beta$-actin in each sample served as a loading control. All data are representative of at least three individual experiments.

Figure 3. PD-1/PD-L1 engagement up-regulates LPS-induced MAPK signaling molecules. 
That said, in a similar pattern to that shown in Figures 1 and 2, not only PD-1 hIg and $\alpha$ PD-L1 antibody treatment but also treatment with hIg and rat IgG isotype control induced the phosphorylation of MAPK molecules, there were no significant differences between groups. Therefore, it seems that when compared to the isotype control, PD-1 hIg and $\alpha$ PD-L1 antibody did not alter the activation pattern of signaling molecules appreciably, and these results suggest that both PD-L1 signaling and IgG-immune complex enhance phosphorylation of MAPK signaling molecules.

\subsection{Induction of Regulatory Macrophages by PD-L1 Signaling in Simulated Physiological Conditions}

Subsequently we considered the possibility that the Fc region of PD-1 hIg or anti PD-L1 antibody may be the cause of increased regulatory macrophage differentiation. To address this hypothesis, we used PD-1 transgenic mice, which overexpress PD-1 protein, particularly in $\mathrm{T}$ cells, as a donor of PD-1 protein to PD-L1 on macrophages to exclude the effect of the Fc region. Splenocytes of wild type or PD-1 Tg mice were treated with mitomycin $\mathrm{C}$ and cocultured with RAW 264.7 cells in various ratios.

Recently, the expression of B7 molecules was found to be enhanced in regulatory macrophages [27]. So, to investigate whether PD-1/PD-L1 interaction signaling altered surface molecules of macrophages, B7-1/B7-2 expression was detected by flow cytometry (Figure 4). When PD-L1 signaling was transferred into the macrophages by PD-1 Tg splenocyte, B7-2 (CD86) level was also up-regulated by PD-L1 signaling, but the expression of B7-1 (also called CD80) was unchanged. In addition, PD-L1, the first step of PD-L1 signaling, was not altered by this signal transduction. Thus, these results suggest that PD-L1 signaling could induce differentiation into regulatory macrophage subsets without the support of Fc receptors.

As the PD-L1 signaling effect induced by PD-1 Tgsplenocytes in regulatory macrophage differentiation was similar in 0.4:1 - 10:1 ratios, we investigated cytokine production in $0.1: 1$ and $0.5: 1$ ratios (Figure 5). The production of the anti-inflammatory cytokine IL-10 was more increased by coculture with PD-1 Tg T cells than by WT T cells, while production of the pro-inflammatory cytokine IL-6 was decreased in both ratios. However, production and secretion of TNF or NO was not altered by PD-1 Tg or WT T cell coculture. To ascertain whether or not mitomycin C-treated $\mathrm{T}$ cells affect RAW cell proliferation, MTT assay was performed in the same conditions. In comparison to LPS-treated RAW cell, no statistically significant differences were observed in WT or PD-1 Tg T cell groups. Taken together, our data
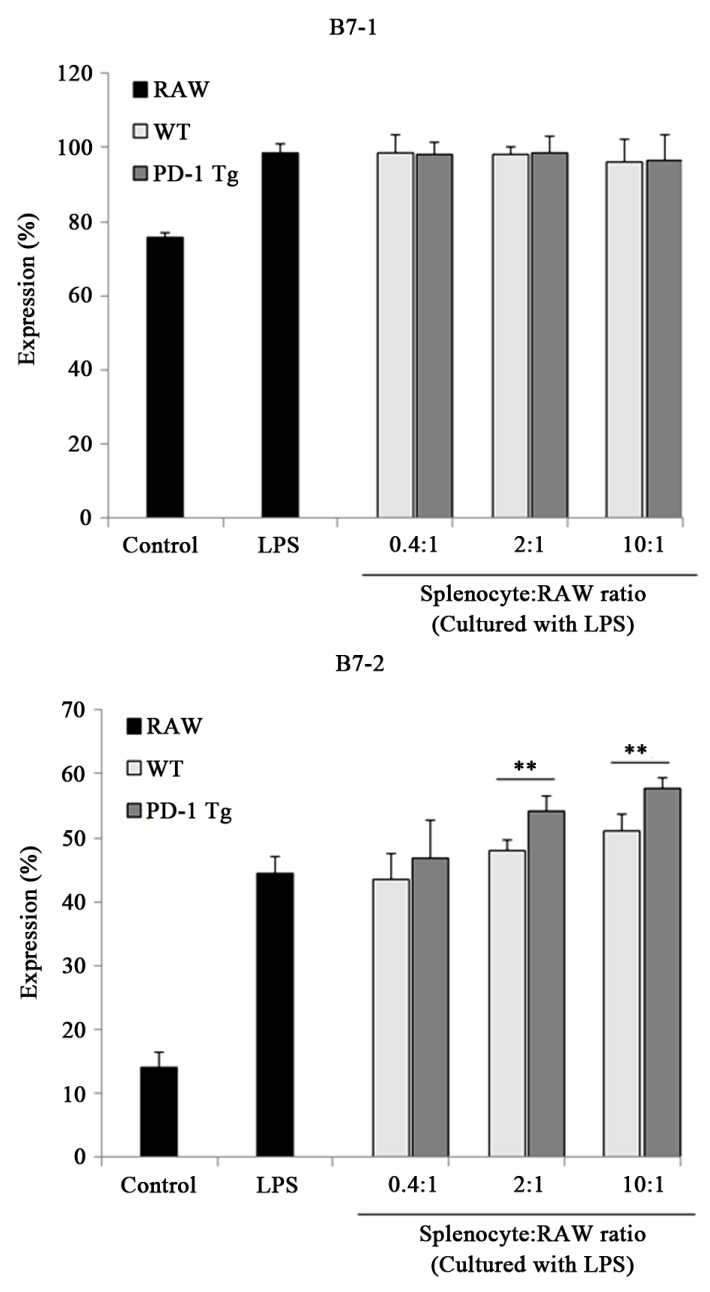

PD-L1

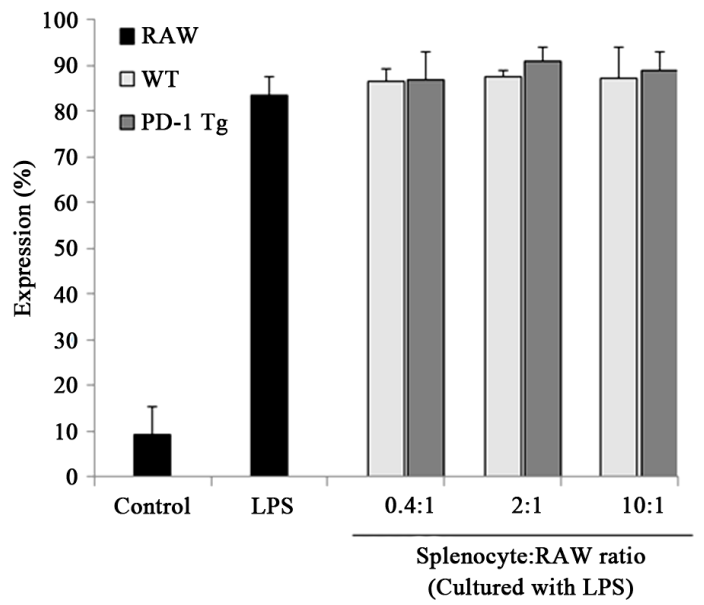

Raw 264.7 cells were cocultured with mitomycin C-treated splenocytes of wild-type or PD-1 transgenic mice in various ratios, $0.4: 1$, 2:1, 10:1 (Splenocyte:RAW cell), for 24 hours in presence of LPS stimulation. CD11b+ RAW cells were stained with PE-conjugated anti-mouse B7-1, B7-2 and PD-L1 antibody for flow cytometry analysis. Student's $t$ test; ${ }^{*} p<0.05 ;{ }^{* *} p<0.01$; and ${ }^{* * *} p<0.001$ vs. WT splenocytes in each ratio. All data are representative of at least three individual experiments.

Figure 4. PD-1 expressed on splenocytes of PD-1 Tg induce alterations of several surface molecules. 

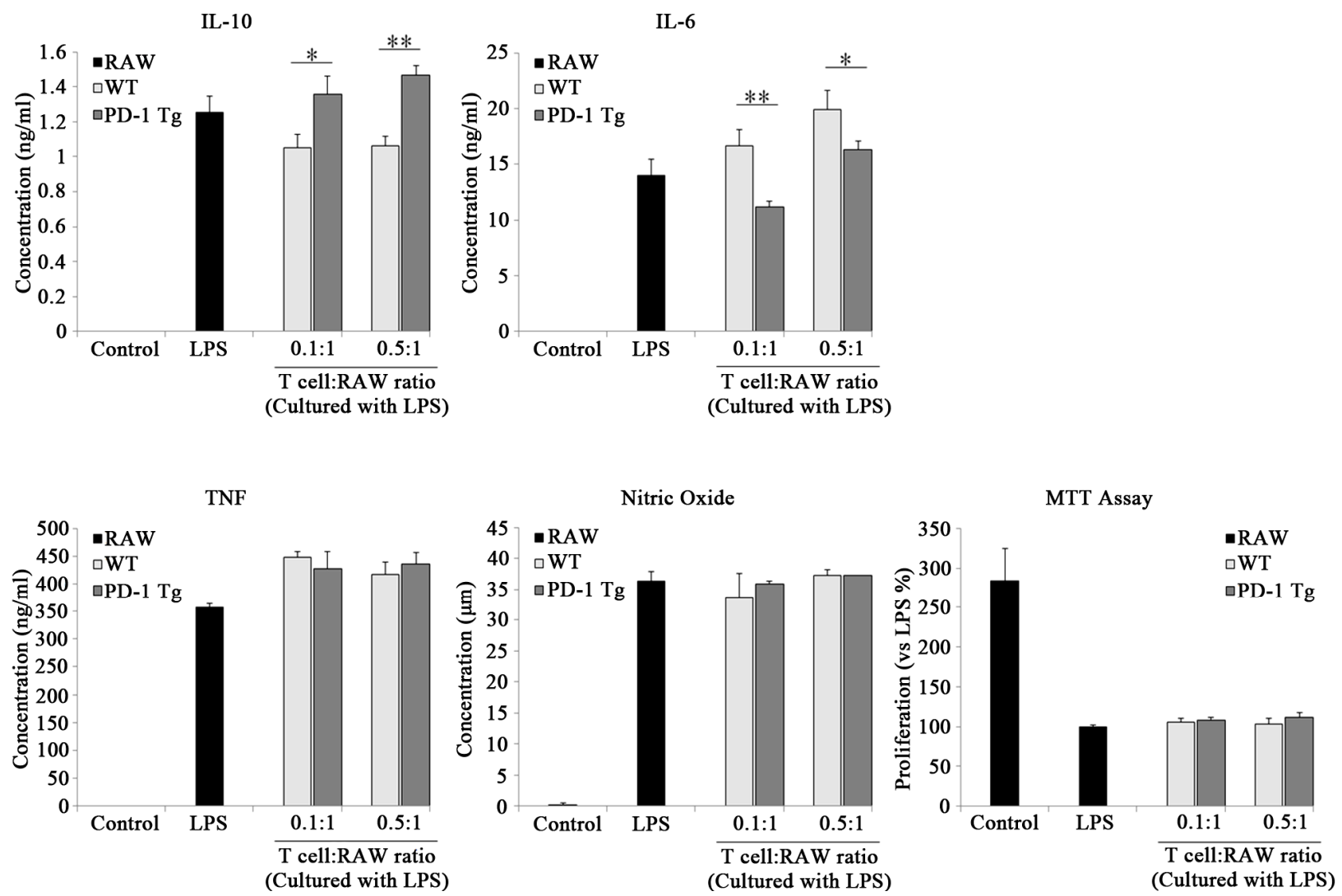

Raw 264.7 cells were cocultured with mitomycin C-treated Thy 1.2+ T cells of wild-type or PD-1 transgenic mice in various ratios, $0.1: 1$, $0.5: 1$ (T cell:RAW cell), in presence of LPS stimulation. After 24 hours, cell free supernatants were harvested and cytokine levels were detected by ELISA; nitric oxide concentration was detected using NO assay. Total cell viability was determined by MTT assay after 24 hours of incubation. Data are presented as mean $\pm \mathrm{SD}$ for triplicate determinations. Student's $t$ test; ${ }^{*} p<0.05 ;{ }^{* *} p<0.01 ;$ and ${ }^{* * *} p<0.001$ vs. WT T cells in each ratio. All data are representative of at least three individual experiments.

Figure 5. PD-1 expressed on T cells of PD-1 Tg turn on the differentiation of regulatory macrophages.

suggest that PD-1/PD-L1 interactions induce differentiation of macrophages towards regulatory subsets and that their functions were similar in the two different studies using PD-1 hIg, anti PD-L1 antibody and using PD-1 molecules expressed on T cells.

\section{DISCUSSION}

Activated macrophages were simply defined as cells that secrete inflammatory mediators and kill intracellular pathogens until a few years ago. Recent studies have shown that activated macrophages may be a more heterogenous group of cells than originally appreciated, with different physiologies and performing distinct immunological functions [28]. Besides the classically activated macrophage lineage which exhibits inflammatory characteristics in host defense, there are two other macrophage subsets. Wound healing macrophages and regulatory macrophages used to be classified as a single cell group-M2 macrophage-but through recent discoveries, this macrophage subset was separated into two distinct subsets [6]. Regulatory macrophages can arise following innate or adaptive immune responses and can inhibit macrophage mediated host defense and inflammatory functions by inhibiting the transcription of proinflammatory cytokine genes and decreasing mRNA stability [29]. The subpopulations of regulatory macrophages have a few differences but there are also common characteristics between them. Most of these regulatory macrophages need at least two stimuli to induce their anti-inflammatory activity. The first signal (such as immune complexes, prostaglandins, adenosine or apoptotic cells) in most cases has little or no stimulatory function on its own; however, when combined with a second stimulus, such as a TLR ligand, the two signals reprogram macrophages into regulatory macrophages [30].

CTLA-4 and CD28, costimulatory molecules similar to PD-1, are known to suppress or stimulate $\mathrm{T}$ cells and macrophages using CTLA-4/B7 and CD28/B7 bi-directional signaling [24,31]. Therefore, we predicted that PD-1/PD-L1 interaction may induce $\mathrm{T}$ cell tolerance and negatively regulate macrophage inflammatory functions. To investigate signaling through PD-L1 on macrophages, PD-1 hIg fusion protein created for the purposes of this study and anti-PD-L1 antibody was used. PD-1 hIg is a 
fusion protein that consists of mouse PD-1 extracellular domain and human IgG1 Fc portion. Both PD-1 hIg and anti-PD-L1 antibody have an affinity to PD-L1 on macrophages. Whether TLR4 stimulation through LPS with PD-L1 signal transduction modulates macrophage activities was confirmed by several experiments.

PD-1 hIg and $\alpha$ PD-L1 antibody with LPS stimulation reduced the pro-inflammatory cytokine IL-6 in mRNA level. Meanwhile, this signaling enhanced the anti-inflammatory cytokine IL-10 and these data were parallel to previously detected IL- 6 and IL-10 patterns at the protein level, but TNF and NO secretions were not changed.

Consequently, it seems that macrophages lost the host defense activity which is associated with pro-inflammatory mediator production, and to infer from the change in IL-10 production, it appears that macrophages took on a regulatory profile through this interaction. Thus, we elaborated on this and conducted follow-up studies to verify how TLR4 stimulation with PD-1/PD-L1 interaction affects the differentiation of regulatory macrophages. Under the same conditions as above, we found that signal transduction through PD-L1 and TLR4 enhanced SPHK1 and LIGHT gene expression, which are distinctive biomarkers of regulatory macrophages.

In this study, PD-1/PD-L1 ligation up-regulated SPHK1 and LIGHT gene expression, and induced high levels of IL-10 but low levels of IL- 6 in the early phases of stimulation. These are all distinct characteristics of regulatory macrophages, and these results serve as evidence to support the hypothesis that PD-1/PD-L1 interactions differentiate macrophages into regulatory subsets.

There are many different ways to generate regulatory macrophages, but solid molecular mechanism that mediates this phenotypic switch has yet to be identified. Out of the few elucidated mechanisms, the most relevant signaling molecules were chosen for confirmation of our hypothesis. D pchejetski et al. reported that LPS-induced TLR4-mediated signaling triggers SPHK1 activation through extracellular signal-regulating kinase, PLC- $1 \gamma$ and PI3 kinase pathways [32]. IL-10 expression can be compromised by inhibition of the p38 signaling pathway in LPS-activated macrophages, and this indicates that there is a critical role for $\mathrm{p} 38$ in IL-10 production [33-35]. These are all signaling molecules that are involved in LPS-induced MAPK pathway signaling, and in this pathway ERK and NF- $\kappa \mathrm{B}$ are also implicated in IL-10 production $[36,37]$.

When this signaling pathway was put under constant LPS stimulation, PD- 1 hIg and $\alpha$ PD-L1 antibody were observed to amplify this signal. However, the isotype control showed similar patterns in phosphorylation of signaling molecules and it was because of the $\mathrm{Fc}$ receptor signaling transduction.
So, to clarify whether the effect of PD-1 hIg and $\alpha \mathrm{PD}-\mathrm{L} 1$ antibody was due to the Fc region, we used PD-1 molecules expressed on $T$ cells directly. In this study, PD-1 Tg and wild type splenocytes or T cells were treated with mitomycin $\mathrm{C}$ and cocultured with RAW cells in various ratios. PD-1 Tg splenocyte enhanced the expression of B7-2 but did not alter the amount of B7-1 or PD-L1. In addition, cytokine secretion from RAW cells cocultured with PD-1 Tg T cells revealed a similar pattern of results to these using PD-1 hIg and $\alpha$ PD-L1 antibody.

Taken together, our data seem to provide evidence for PD-1/PD-L1 engagement inducing a regulatory profile in macrophages. Regulatory macrophages derived from PD-L1 signaling lost their host defense activity, which consists of the production of pro-inflammatory cytokine IL-6 and the exhibition of increased IL-10, SPHK1 and LIGHT levels. This differentiation seemed to occur through excessive activation of MAPK signaling pathways but it was similar to activation of signaling molecules by the isotype control. In a condition that mimics the actual physiological environment, PD-1 expressed on $\mathrm{T}$ cells directly engaged with PD-L1 on macrophages and could deliver comparable signaling and induce differentiation. It can be hypothesized that the decrease in macrophage population observed in type $1 \mathrm{DM}$-induced PD-1 Tg mice described in our previous work might have been caused by overexpression of PD-1 and the subsequent differentiation of macrophages into the regulatory subset. In conclusion, PD-L1 on macrophages is able to deliver signaling through interaction with PD-1 on $T$ cells and this combination induces the differentiation of macrophages into the regulatory subset. Similar to regulatory $\mathrm{T}$ cells, regulatory macrophages can also contribute to controlling an overactived immune system. Further studies are needed, as the understanding and modulating of regulatory macrophages may lead to new approaches for treatment or prevention of auto-immune diseases, such as type I diabetes, rheumatic syndrome and hypersensitivity-related diseases, which are concerned with the over production of inflammatory cytokines in macrophages.

\section{ACKNOWLEDGEMENTS}

This research was supported by the Chung-Ang University Excellent Student Scholarship and Basic Science Research Program through the National Research Foundation of Korea (NRF) funded by the Ministry of Education, Science and Technology (2010-0012877).

\section{REFERENCES}

[1] Montano, A.M., Tsujino, F., Takahata, N. and Satta, Y. (2011) Evolutionary origin of peptidoglycan recognition proteins in vertebrate innate immune system. BMC Evo- 
lutionary Biology, 11, 79. doi:10.1186/1471-2148-11-79

[2] Auger, M.J. and Ross, J.A. (1993) The biology of the macrophage. In: Lewis, C.E. and McGee, J.O'D., Eds., The Macrophage, Oxford University Press, Oxford, 1-74.

[3] Brem-Exner, B.G., Sattler, C., Hutchinson, J.A., Koehl, K. Kronenberg, G.E., Farkas, S., Inoue, S., Blank, C., Knechtle, S.J., Schlitt, H.J., Fandrich, F. and Geissler, E.K. (2008) Macrophages driven to a novel state of activation have anti-inflammatory properties in mice. Journal of Immunology, 180, 335-349.

[4] Stout, R.D. and Suttles, J. (2004) Functional plasticity of macrophages: Reversible adaptation to changing microenvironments. Journal of Leukocyte Biology, 76, 509513. doi: $10.1189 / \mathrm{jlb} .0504272$

[5] Anderson, C.F. and Mosser, D.M. (2002) Cutting edge: Biasing immune responses by directing antigen to macrophage Fc gamma receptors. Journal of Immunology, 168, 3697-3701.

[6] Mosser, D.M. and Edwards, J.P. (2008) Exploring the full spectrum of macrophage activation. Nature Reviews Immunology, 8, 958-969. doi:10.1038/nri2448

[7] Zhang, X. and Mosser, D.M. (2008) Macrophage activation by endogenous danger signals. Journal of Pathology, 214, 161-178. doi:10.1002/path.2284

[8] Stout, R.D., Jiang, C., Matta, B., Tietzel, I., Watkins, S.K. and Suttles, J. (2005) Macrophages sequentially change their functional phenotype in response to changes in microenvironmental influences. Journal of Immunology, 175, 342-349.

[9] Sharpe, A.H. and Freeman, G.J. (2002) The B7-CD28 superfamily. Nature Reviews Immunology, 2, 116-126. doi: $10.1038 /$ nri727

[10] Chen, L., Hussien, Y., Hwang, K.W., Wang, Y., Zhou, P. and Alegre, M.L. (2008) Overexpression of program death-1 in T cells has mild impact on allograft survival. Transplant International, 21, 21-29.

[11] Ishida, Y., Agata, Y., Shibahara, K. and Honjo, T. (1992) Induced expression of PD-1, a novel member of the immunoglobulin gene superfamily, upon programmed cell death. EMBO Journal, 11, 3887-3895.

[12] Agata, Y., Kawasaki, A., Nishimura, H., Ishida, Y., Tsubata, T., Yagita, H. and Honjo, T. (1996) Expression of the PD-1 antigen on the surface of stimulated mouse T and B lymphocytes. International Immunology, 8, 765772. doi:10.1093/intimm/8.5.765

[13] Nishimura, H., Agata, Y., Kawasaki, A., Sato, M., Imamura, S., Minato, N., Yagita, H., Nakano, T. and Honjo, T. (1996) Developmentally regulated expression of the PD-1 protein on the surface of double-negative (CD4CD8-) thymocytes. International Immunology, 8, 773780. doi:10.1093/intimm/8.5.773

[14] del Rio, M.L., Lucas, C.L., Buhler, L., Rayat, G. and Rodriguez-Barbosa, J.I. (2010) HVEM/LIGHT/BTLA/ CD160 cosignaling pathways as targets for immune regulation. Journal of Leukocyte Biology, 87, 223-235. doi:10.1189/jlb.0809590

[15] Dong, H., Zhu, G., Tamada, K. and Chen, L. (1999) B7$\mathrm{H} 1$, a third member of the B7 family, co-stimulates T- cell proliferation and interleukin-10 secretion. Nature Medicine, 5, 1365-1369. doi:10.1038/70932

[16] Freeman, G.J., Long, A.J., Iwai, Y., Bourque, K., Chernova, T., Nishimura, H., Fitz, L.J., Malenkovich, N., Okazaki, T., Byrne, M.C., Horton, H.F., Fouser, L., Carter, L., Ling, V., Bowman, M.R., Carreno, B.M., Collins, M., Wood, C.R. and Honjo, T. (2000) Engagement of the PD-1 immunoinhibitory receptor by a novel B7 family member leads to negative regulation of lymphocyte activation. Journal of Experimental Medicine, 192, 1027 1034. doi:10.1084/jem.192.7.1027

[17] Latchman, Y., Wood, C.R., Chernova, T., Chaudhary, D., Borde, M., Chernova, I., Iwai, Y., Long, A.J., Brown, J.A., Nunes, R., Greenfield, E.A., Bourque, K., Boussiotis, V.A., Carter, L.L., Carreno, B.M., Malenkovich, N., Nishimura, H., Okazaki, T., Honjo, T., Sharpe, A.H. and Freeman, G.J. (2001) PD-L2 is a second ligand for PD-1 and inhibits $\mathrm{T}$ cell activation. Nature Immunology, 2, 261-268. doi:10.1038/85330

[18] Yamazaki, T., Akiba, H., Iwai, H., Matsuda, H., Aoki, M., Tanno, Y., Shin, T., Tsuchiya, H., Pardoll, D.M., Okumura, K., Azuma, M. and Yagita, H. (2002) Expression of programmed death 1 ligands by murine $\mathrm{T}$ cells and APC. Journal of Immunology, 169, 5538-5545.

[19] Nishimura, H., Minato, N., Nakano, T. and Honjo, T. (1998) Immunological studies on PD-1 deficient mice: Implication of PD-1 as a negative regulator for B cell responses. International Immunology, 10, 1563-1572. doi:10.1093/intimm/10.10.1563

[20] Nishimura, H., Honjo, T. and Minato, N. (2000) Facilitation of beta selection and modification of positive selection in the thymus of PD-1-deficient mice. Journal of Experimental Medicine, 191, 891-898. doi:10.1084/jem.191.5.891

[21] Blazar, B.R., Taylor, P.A., Panoskaltsis-Mortari, A., Sharpe, A.H. and Vallera, D.A. (1999) Opposing roles of CD28:B7 and CTLA-4:B7 pathways in regulating in vivo alloresponses in murine recipients of MHC disparate $\mathrm{T}$ cells. Journal of Immunology, 162, 6368-6377.

[22] Levine, B.L., Ueda, Y., Craighead, N., Huang, M.L. and June, C.H. (1995) CD28 ligands CD80 (B7-1) and CD86 (B7-2) induce long-term autocrine growth of CD4+ T cells and induce similar patterns of cytokine secretion in vitro. International Immunology, 7, 891-904.

doi:10.1093/intimm/7.6.891

[23] Salomon, B. and Bluestone, J.A. (2001) Complexities of CD28/B7: CTLA-4 costimulatory pathways in autoimmunity and transplantation. Annual Review of Immunology, 19, 225-252. doi:10.1146/annurev.immunol.19.1.225

[24] Orabona, C., Grohmann, U., Belladonna, M.L., Fallarino, F., Vacca, C., Bianchi, R., Bozza, S., Volpi, C., Salomon, B.L., Fioretti, M.C., Romani, L. and Puccetti, P. (2004) CD28 induces immunostimulatory signals in dendritic cells via CD80 and CD86. Nature Immunology, 5, 1134 1142. doi: $10.1038 /$ ni1124

[25] Won, T.J., Jung, Y.J., Kwon, S.J., Lee, Y.J., Lee do, I., Min, H., Park, E.S., Joo, S.S. and Hwang, K.W. (2010) Forced expression of programmed death-1 gene on T cell decreased the incidence of type 1 diabetes. Archives of Pharmacal Research, 33, 1825-1833. 
doi:10.1007/s12272-010-1115-3

[26] Teng, M.W., Swann, J.B., Koebel, C.M., Schreiber, R.D. and Smyth, M.J. (2008) Immune-mediated dormancy: An equilibrium with cancer. Journal of Leukocyte Biology, 84, 988-993. doi:10.1189/jlb.1107774

[27] Fleming, B.D. and Mosser, D.M. (2011) Regulatory macrophages: Setting the threshold for therapy. European Journal of Immunology, 41, 2498-2502. doi:10.1002/eji.201141717

[28] Mosser, D.M. (2003) The many faces of macrophage activation. Journal of Leukocyte Biology, 73, 209-212. doi:10.1189/jlb.0602325

[29] Sternberg, E.M. (2006) Neural regulation of innate immunity: A coordinated nonspecific host response to pathogens. Nature Reviews Immunology, 6, 318-328. doi:10.1038/nri1810

[30] Yi, A.K., Yoon, J.G., Yeo, S.J., Hong, S.C., English, B.K. and Krieg, A.M. (2002) Role of mitogen-activated protein kinases in CpG DNA-mediated IL-10 and IL-12 production: Central role of extracellular signal-regulated kinase in the negative feedback loop of the CpG DNAmediated Th1 response. Journal of Immunology, 168, 4711-4720.

[31] Grohmann, U., Orabona, C., Fallarino, F., Vacca, C., Calcinaro, F., Falorni, A., Candeloro, P., Belladonna, M.L., Bianchi, R., Fioretti, M.C. and Puccetti, P. (2002) CTLA4-Ig regulates tryptophan catabolism in vivo. Nature Immunology, 3, 1097-1101. doi:10.1038/ni846

[32] Pchejetski, D., Nunes, J., Coughlan, K., Lall, H., Pitson, J., Waxman, S.M. and Sumbayev, V.V. (2011) The involvement of sphingosine kinase 1 in LPS-induced Toll- like receptor 4-mediated accumulation of HIF-1 alpha protein, activation of ASK1 and production of the proinflammatory cytokine IL-6. Immunology and Cell Biology, 89, 268-274. doi:10.1038/icb.2010.91

[33] Ma, W., Lim, W., Gee, K., Aucoin, S., Nandan, D., Kozlowski, M., Diaz-Mitoma, F. and Kumar, A. (2001) The p38 mitogen-activated kinase pathway regulates the human interleukin-10 promoter via the activation of $\mathrm{Sp} 1$ transcription factor in lipopolysaccharide-stimulated human macrophages. Journal of Biological Chemistry, 276, 13664-13674.

[34] Okazaki, T., Iwai, Y. and Honjo, T. (2002) New regulatory co-receptors: Inducible co-stimulator and PD-1. $\mathrm{Cu}$ rrent Opinion in Immunology, 14, 779-782. doi:10.1016/S0952-7915(02)00398-9

[35] Park, J.M., Greten, F.R., Wong, A., Westrick, R.J., Arthur, J.S., Otsu, K., Hoffmann, A., Montminy, M. and Karin, M. (2005) Signaling pathways and genes that inhibit pathogen-induced macrophage apoptosis-CREB and NF-kappaB as key regulators. Immunity, 23, 319-329. doi:10.1016/j.immuni.2005.08.010

[36] Lucas, M., Zhang, X., Prasanna, V. and Mosser, D.M. (2005) ERK activation following macrophage FcgammaR ligation leads to chromatin modifications at the IL-10 locus. Journal of Immunology, 175, 469-477.

[37] Slack, E.C., Robinson, M.J., Hernanz-Falcon, P., Brown, G.D., Williams, D.L., Schweighoffer, E., Tybulewicz, V.L. and Reis e Sousa, C. (2007) Syk-dependent ERK activation regulates IL-2 and IL-10 production by DC stimlated with zymosan. European Journal of Immunology, 37, 1600-1612. doi:10.1002/eji.200636830 